\title{
Introduction to Reports from the Field Minitrack
}

\author{
Julee Hafner \\ The Chicago School Of Professional Psychology \\ haf2lead@gmail.com
}

\author{
Vincent Scovetta \\ Long Island University \\ Vincent.Scovetta@1iu.edu
}

\begin{abstract}
Welcome to this, the 51st anniversary of the Hawaii International Conference on System Sciences (HICSS) conferences. This is the fourth year of the Reports from the Field Minitrack, proudly bringing you the latest research focused on application of knowledge management issues as reported by both researchers and practitioners alike.
\end{abstract}

\section{Introduction}

Knowledge Management theory and associated research continue to expand. There are many journals that focus on KM topics in information systemsoriented research. Knowledge workers, who develop, implement, manage, and use knowledge management systems are of interest. The gap in the knowledge between the knowledge systems research currently being conducted, and the needs and experiences of knowledge workers is real.

This minitrack is devoted to KM theory and its application from a practitioner perspective. Here in this minitrack, Reports From The Field, is devoted to providing academic scholars that conduct research in building theory while improving the application of knowledge management systems in the practitioner community.

The practitioners in the field who actually create, store, retrieve, and use the knowledge contained in a knowledge management system can provide invaluable insight to the research community in terms of practical application skills. Similarly, well-directed research can enhance the practice of knowledge management.

While other types of papers are welcomed, a single case study approach is generally of greatest interest in this minitrack. It is hoped that accepted submissions will document specific knowledge system successes or failures. Since the minitrack emphasis is on experiences and collected information from the practitioner community and application of study, the traditional scholarly requirements for the conference are somewhat softened.

\section{Shellon Blackman-Lees}

Our first presenter is Shellon Blackman- Lees who will present a paper entitled: Towards a Conceptual Framework for Persistent Use: A Technical Plan to Achieve Semantic Interoperability within Electronic Health Record Systems.

In the healthcare system, patient data is transmitted to various sources in completing patient care. It is essential that semantic interoperability be considered as Electronic Health Record (EHR) to improve adoption. Reluctance by the healthcare industry to implement these EHRs for the purpose of achieving interoperability has led to concern that adoption and functional use are lagging behind. Currently, no existing single data standardization structure that can effectively share and interpret patient data within heterogeneous systems exists.

The current research used the design science research methodology (DSRM) to design and develop a master data standardization and translation (MDST) model which incorporated the Resource Description Framework (RDF). Using RDF, a universal exchange language, may provide the solution to this problem.

\section{Fabian Utz, Christian Newmann, and Tafreschi Omid}

Our next presenters will be How to Discover Fabian Utz, Christian Neumann, and Tafreschi Omid who will present their submission, Knowledge for Improving Availability in the Manufacturing Domain?

This paper uses a specific process model for Knowledge Discovery in Databases (KDD) projects. The goal is to improve manufacturing processes. For this purpose, Overall Equipment Efficiency (OEE) is 
analyzed and used, since it is an approved approach to monitor and improve the degree of availability in manufacturing. To define the specific process model, the generic CRISP-DM reference model was used to conduct mapping for availability improvement. The applicability of the model is demonstrated in the context of a specific KDD project in a large enterprise in the manufacturing industry.

\section{Alexander Kaiser,Florian Fahrenbach, Florian Kragulj, and Thomas Grisold}

These authors, for our third paper, will present, Towards a prioritization of needs to support decision making in organizational change processes.

They introduce a prioritization of needs decision support system within an organization. Boundary critique, is used to identify critical stakeholders that lead to three different viewpoints in the decision support system. These viewpoints are focused on internal, external and systemic viewpoints and that allow for assessment and discovery of interrelationships between organizational stakeholders.

Assessment of stakeholder views are examined by different dimensions, are subsequently combined, and weighted. Based on the identification and examination of these needs, an index is determined. An organizational case study conducted in Austria allows for the application of this index, as well as implications for theory and practice will be discussed.

\section{Lina Lo}

Our final presentation will come from Lina Lo who will present her paper, Understanding Knowledge Flow Dynamics During the Reimplementation Phase of an Enterprise Resource Planning Project. Building on her previous work, Lina uses knowledge-flow theory to suggest critical factors for enterprise resource planning within the context of real life immersion case.

\section{Conclusion}

We wish to thank each of our presenters for their research and willingness to share their results with us during another year of celebration at HICSS-51. We look forward seeing new research studies that contribute to application of $\mathrm{KM}$ in business and industry. We hope researchers will consider contributing to our mini-track Reports from the Field next year. 